\section{Progressive ocular inflammation following anti- tubercular therapy for presumed ocular tuberculosis in a high-endemic setting}

S Basu, S Nayak, TR Padhi and T Das

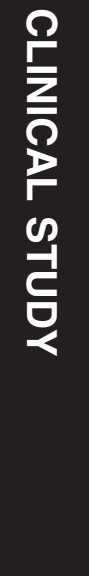

vitrectomy for resolution. Three patients $\mathbf{( 1 1 . 5 \% ) ~ h a d ~ p e r s i s t e n t ~ i n f l a m m a t i o n ~ a t ~ e n d ~ o f ~}$ follow-up period.

Conclusion Progressive inflammation following ATT for presumed ocular TB is common. It generally resolves on escalation of corticosteroid therapy. Cases not responding to increased immunosuppression need to be re-investigated to rule out a nontubercular cause.

Eye (2013) 27, 657-662; doi:10.1038/eye.2013.5; published online 1 March 2013

Keywords: inflammation; tuberculosis; ocular; anti-tubercular therapy; progressive inflammation

\section{Introduction}

One-third of the world's population is infected with Mycobacterium tuberculosis and can potentially manifest features of ocular tuberculosis (TB). ${ }^{1}$ Not surprisingly, TB accounts for large proportion of infectious uveitis cases in endemic countries. ${ }^{2}$ The benefits of anti-tubercular therapy (ATT) in treatment of TB-associated uveitis are well documented. ${ }^{3}$ Several clinical indicators of ocular TB (applicable in high-endemic settings) have also been identified based on the response to ATT. ${ }^{4}$

However, in most cases, the diagnosis of ocular TB remains presumptive because of lack of definitive (microscopy, culture, or PCR) evidence. In such situations, progressive ocular inflammation following ATT can pose a serious diagnostic challenge for the treating physician. It
L V Prasad Eye Institute, Bhubaneswar, India

Correspondence: S Basu, L V Prasad Eye Institute, Patia, India.

Tel: + 916743989 202;

Fax: + 916743987130 .

E-mail: eyetalk@gmail.com Accepted in revised form: 26 December 2012 Published online: 1 March 2013 Bhubaneswar 751024 ,

Received: 20 April 2012 
can be attributed to missed diagnosis, drug resistance, reinfection, or immunological response to tubercular antigens released during ATT-each requiring a different approach to management. Although an alternative diagnosis or drug resistance may need change in specific anti-microbial therapy, immunological reaction to tubercular antigens is usually managed by escalating corticosteroid therapy.

Previous studies on role of ATT in ocular TB do not provide detailed analysis of such cases of progressive ocular inflammation. A large study from India (216 patients) mentions that $15.74 \%$ (nearly one in six) patients developed recurrent inflammation despite ATT. ${ }^{3}$ The anatomic location of uveitis and tuberculin reactivity in these patients has been mentioned. However, the clinical details, time of recurrence, and further course of management have not been described. More recently, continued progression after ATT has been described in patients with tuberculous serpiginous-like choroiditis (SLC). ${ }^{5}$ There are no large studies describing such progressive inflammation in other forms of ocular TB. In the present study, we have further analyzed the incidence, clinical presentation, revised diagnoses, and response to treatment in patients who developed progressive ocular inflammation following ATT for presumed ocular TB.

\section{Materials and methods}

We retrospectively analyzed medical records of all patients who were diagnosed as presumed ocular TB and advised ATT between 1 April 2008 and 15 June 2010 at our center, a tertiary care facility in eastern India. All patients were of South Asian ethnicity and were identified by a disease register maintained in the uveitis clinic. We included patients who were initiated on ATT and completed a minimum of 1-year follow-up after initiation of such therapy.

All patients had a detailed recording of history including contact with known TB patients. A complete examination was done including visual acuity, applanation tonometry, slit lamp biomicroscopy, and dilated fundus evaluation. Standardization of uveitis nomenclature working group recommendations were used for anatomical classification of uveitis and grading of anterior chamber inflammation. ${ }^{6}$ The National Eye Institute system was adopted for grading vitreous haze. Ancillary tests like fluorescein angiography, optical coherence tomography, B-scan ultrasonography, and ultrasound biomicroscopy were done as required. Systemic evaluation and laboratory investigations were guided by the pattern of uveitis identified on historytaking and ocular examination, though baseline investigations like complete blood counts, erythrocyte sedimentation rate, tuberculin test, and chest $\mathrm{X}$-ray were done for all patients. Whenever TB was suspected as a possible etiology, special attention was paid to detection of other foci of extra-pulmonary TB.

The diagnosis of presumed ocular TB was based on previously published guidelines. ${ }^{3}$ These included the presence of clinical signs consistent with ocular TB, associated with any one or more of the following: (1) positive tuberculin reaction (induration $>10 \mathrm{~mm}$, with dosage of five tuberculin units) or interferon-gamma release assay; (2) evidence of active/healed pulmonary/ extra-pulmonary TB; (3) positive therapeutic response to four-drug ATT for 4-6 weeks; and (4) exclusion of other uveitis entities (infectious and noninfectious), which mimic the given clinical presentation of ocular TB. The cut off of $10 \mathrm{~mm}$ for the tuberculin test was based on the guidelines from Center of Disease Control, Atlanta, which has recommended this value for high-prevalence countries such as India. ${ }^{7}$ In general, these guidelines recommend lower cut off limits for populations with higher pre-test probability of the disease. All patients with a positive tuberculin reaction or interferon-gamma release assay were also reviewed by an internist, before initiation of ATT.

We treated all presumed ocular TB patients with a combination of ATT and corticosteroid therapy. ATT consisted of isoniazid $5 \mathrm{mg} / \mathrm{kg} /$ day, rifampicin $450 \mathrm{mg} /$ day if body weight was $\leq 50 \mathrm{~kg}$ and $600 \mathrm{mg} /$ day if body weight was $>50 \mathrm{~kg}$, ethambutol $15 \mathrm{mg} / \mathrm{kg} /$ day, and pyrazinamide $25-30 \mathrm{mg} / \mathrm{kg} /$ day for initial 2 months. Thereafter, rifampicin and isoniazid were used for another 4 months. The total duration of therapy was restricted to 6 months, based on the World Health Organization (WHO) recommendations for extra-pulmonary TB. ${ }^{8}$ All patients were carefully monitored for compliance to ATT and drug toxicity by the treating internist. The routes of administration of corticosteroid therapy were based on the anatomical location of uveitis, whereas its dosage and duration depended on the degree of intraocular inflammation. In general, topical ( \pm periocular) corticosteroids were preferred for anterior uveitis, periocular ( \pm systemic) corticosteroids for intermediate uveitis, and oral ( \pm intra-venous) corticosteroids for posterior and pan-uveitis. The starting dosage of oral corticosteroids (prednisolone), when required, was $1 \mathrm{mg} /$ $\mathrm{kg}$ body in all patients. Systemic immunosuppressives were used whenever deemed necessary.

The primary outcome measure was progression (worsening) of inflammation, defined as a two-step increase in level of inflammation (anterior chamber/ vitreous) or the appearance of new lesions (eg, retinal vasculitis, choroidits, and optic neuritis) following initiation of ATT. Whenever such progression was noted, detailed ocular and systemic evaluation (including a 
repeat or new laboratory investigations, if required) was done to rule out any alternative diagnosis. If necessary, pulmonologist opinion was sought to rule out drug resistance. Where our working diagnosis remained presumed ocular TB, we escalated corticosteroid dosage (with/without additional systemic immunosuppressives), without discontinuing ATT. If an alternative diagnosis was considered, specific therapy was started for the given uveitis entity (eg, antibiotics for infectious uveitis).

The following information was collected from patient records: patient's age, gender, disease laterality, presence of anterior chamber cells, flare, iris nodules, synechiae (anterior and posterior), lens status, vitreous cells, snowballs, pars plana exudates (snow-banking), retinal vasculitis, choroiditis (focal/multifocal/serpiginouslike), sub-retinal abscess, neuro-retinitis, optic neuritis, episcleritis, and scleritis. Because tubercular SLC is often multifocal, we termed superficial lesions with ameboid margins as SLC and deeper choroidal lesions as multifocal choroiditis. Details of treatment including dosage, duration, and routes of administration of corticosteroids and immunosuppressives, and the therapeutic response (quiescence or worsening/ progression of inflammation) were noted. In cases of worsening/progression, the current dose of corticosteroids, any revision in diagnosis, change in treatment plan, and outcome of such treatment were documented. Descriptive statistics like mean, median, $\mathrm{SD}$, and range were used to evaluate the study population.

\section{Results}

In all, 134 out of 147 patients $(n=134 ; 91.2 \%)$ agreed for ATT; 26 patients (19.4\%) did not complete 1-year followup. Two patients discontinued treatment because of drug intolerance. Of the remaining 106 patients, 64 were male and 42 were female. The median age of these patients was 33.5 years (range 12-60 years). The median followup period was 20 months (range 12.5-35 months).

Fifty-five (51.9\%) patients had bilateral disease. Healed or active pulmonary TB (including hilar/mediastinal lymphadenopathy) was noted in $23(21.7 \%)$ patients, and extra-pulmonary TB was seen in $3(2.8 \%)$ patients. Focal lesions (healed or active chorioretinitis/choroiditis) were seen in more than half ( 33 out of $60,55 \%$ ) of the patients with anterior or intermediate uveitis or retinal vasculitis. The demographic details of patients in each clinical category of uveitis are provided in Table 1.

In all, progressive intraocular inflammation after initiation of ATT was seen in $26(24.5 \%)$ patients, the largest proportion $(37 \%, 9$ out of 27$)$ being in those with intermediate uveitis (Table 2). The median time of onset of inflammation varied from 0.5 months in patients with SLC and multifocal choroiditis to 3.5 months in case of intermediate uveitis. In 22 patients $(85.2 \%)$, the site of progressive inflammation was the same as presenting site. But in four cases, inflammatory signs were noted at a site different from the original one. This included one patient with unilateral granulomatous anterior uveitis who later developed bilateral multifocal choroiditis, one patient each of intermediate uveitis and SLC, who

Table 1 Demographic details of patients with presumed ocular tuberculosis

\begin{tabular}{|c|c|c|c|c|c|c|}
\hline & $\begin{array}{l}\text { Anterior } \\
\text { uveitis }\end{array}$ & $\begin{array}{l}\text { Intermediate } \\
\text { uveitis }\end{array}$ & $\begin{array}{l}\text { Retinal } \\
\text { vasculitis }\end{array}$ & $\begin{array}{c}\text { Serpiginous- } \\
\text { like } \\
\text { choroiditis }\end{array}$ & $\begin{array}{c}\text { Multifocall } \\
\text { focal } \\
\text { choroiditis }\end{array}$ & Pan-uveitis \\
\hline Total patients & 7 & 27 & 26 & 24 & 9 & 13 \\
\hline Median age and range (in years) & $35(27-42)$ & $39(16-58)$ & $26(12-52)$ & $33(16-60)$ & $27(22-46)$ & $38(19-58)$ \\
\hline \multicolumn{7}{|l|}{ Gender } \\
\hline Male & 2 & 10 & 24 & 20 & 4 & 4 \\
\hline Female & 5 & 17 & 2 & 4 & 5 & 9 \\
\hline \multicolumn{7}{|l|}{ Laterality } \\
\hline Bilateral & 3 & 18 & 18 & 9 & 3 & 4 \\
\hline Unilateral & 4 & 9 & 8 & 15 & 6 & 9 \\
\hline Pulmonary TB (healed/active) & 1 & 7 & 5 & 2 & 4 & 4 \\
\hline $\begin{array}{l}\text { Associated focal chorio-retinal } \\
\text { lesions (active/inactive) }\end{array}$ & 2 & 8 & 23 & NA & NA & NA \\
\hline \multicolumn{7}{|l|}{ Initial treatment } \\
\hline ATT + local steroid & - & - & 2 & - & 2 & - \\
\hline ATT + systemic & 7 & 12 & - & - & - & 13 \\
\hline Steroid & - & 15 & 24 & 24 & 7 & \\
\hline
\end{tabular}


Table 2 Clinical details for patients presenting with progressive ocular inflammation in each clinical category of presumed ocular tuberculosis

\begin{tabular}{|c|c|c|c|c|c|c|}
\hline & $\begin{array}{l}\text { Anterior } \\
\text { uveitis } \\
(\mathrm{n}=7)\end{array}$ & $\begin{array}{c}\text { Intermediate } \\
\text { uveitis }(\mathrm{n}=27)\end{array}$ & $\begin{array}{c}\text { Retinal } \\
\text { vasculitis } \\
(\mathrm{n}=26)\end{array}$ & $\begin{array}{c}\text { Serpiginous-like } \\
\text { choroiditis }(\mathrm{n}=24)\end{array}$ & $\begin{array}{c}\text { Multifocal/ focal } \\
\text { choroiditis }(\mathrm{n}=9)\end{array}$ & $\begin{array}{l}\text { Pan-uveitis } \\
\quad(\mathrm{n}=13)\end{array}$ \\
\hline $\begin{array}{l}\text { Number of patients who developed } \\
\text { progressive inflammation }\end{array}$ & 1 & 9 & 3 & 7 & 2 & 4 \\
\hline $\begin{array}{l}\text { Median time of onset of progression (in } \\
\text { months, range) }\end{array}$ & 2 & $3.5(1.5-14)$ & $1.0(0.5-14)$ & $0.5(0.25-5)$ & 0.5 & $0.75(0.5-1.5)$ \\
\hline \multicolumn{7}{|l|}{ Site of progressive inflammation } \\
\hline Same as presenting site & - & 8 & 2 & 6 & 2 & 4 \\
\hline Different from presenting site & 1 & 1 & 1 & 1 & 0 & - \\
\hline \multicolumn{7}{|l|}{ Post-progression diagnosis } \\
\hline Same as initial diagnosis (ocular TB) & 1 & 2 & 2 & 7 & 2 & 2 \\
\hline Alternative diagnosis & & 1 & 1 & - & - & - \\
\hline Undetermined & - & 6 & - & - & - & 2 \\
\hline \multicolumn{7}{|l|}{ Status at last visit } \\
\hline $\begin{array}{l}\text { Resolved with increased } \\
\text { corticosteroids }\end{array}$ & 1 & 2 & 2 & 7 & 2 & 2 \\
\hline Resolved with alternative therapy & - & 6 & 1 & - & - & - \\
\hline Persistent inflammation & - & 1 & - & - & - & 2 \\
\hline
\end{tabular}

developed episcleritis, and one patient with retinal vasculitis who developed optic neuritis. All the four cases resolved on escalating corticosteroid therapy. Of the remaining 22 patients (progressive inflammation at the same site as at presentation), the original diagnosis was retained in majority $(63.6 \%, n=16)$ of patients, all of who responded to escalation in corticosteroid dosage.

However, only two out of nine patients with intermediate uveitis who worsened after initiating ATT responded to corticosteroid escalation. The diagnosis remained undetermined in six out of nine patients, five of whom resolved with therapeutic vitrectomy. Notably, none of these six patients had associated focal chorioretinal lesions, healed or active. One patient, who declined surgery, had persistent inflammation till last follow-up.

The diagnosis was revised in two patients following onset of progressive inflammation. One patient with intermediate uveitis was diagnosed as Lyme's disease and responded to oral doxycycline therapy. Another patient with retinal vasculitis was later diagnosed as Behcet's uveitis, and required aggressive immunosuppressive therapy.

\section{Discussion}

Our study describes the spectrum of progressive ocular inflammation following initiation of ATT for various clinical manifestations of presumed ocular TB.
It highlights the challenges faced by the treating physician in a high-endemic country while managing such cases. The likelihood of TB-associated uveitis is high in these countries, and so is the possibility of falsepositive diagnosis. The problem is compounded further by the variety of factors that can affect the treatment outcome, following ATT.

Intermediate uveitis, retinal vasculitis, and SLC (in that order) were the most common clinical manifestations of presumed ocular TB in our study. Significantly, anterior uveitis accounted for only $6.6 \%$ cases, as against $31.5 \%$ (68 of 216 cases) in an earlier series. ${ }^{3}$ Healed or active pulmonary TB (including hilar lymphadenopathy) was detected in only $21.7 \%$ patients, whereas extrapulmonary TB was seen in $2.8 \%$. It is possible that more sensitive investigations like high-resolution computed tomography or positron emission tomography would have detected more systemic TB cases. ${ }^{9}$ Almost a quarter $(24.5 \%)$ of our patients developed progressive ocular inflammation following initiation of ATT. This included 16 patients $(15.1 \%)$ who improved with escalation of corticosteroid therapy and 10 patients $(9.4 \%)$ in whom the diagnosis was changed or remained undetermined. An earlier study by Bansal et $a l^{3}$ had reported an overall recurrence rate of $15.7 \%$ after initiation of ATT for TB-associated uveitis. However, the cause of these recurrences had not been evaluated in this study.

In the present study, progressive inflammation associated with most forms of presumed ocular TB 
(except intermediate uveitis) appeared to resolve on escalation of corticosteroid therapy. We believe that these cases represent paradoxical worsening (JarischHerxheimer reaction), which has been reported following initiation of chemotherapy for TB and several other infections. ${ }^{5}$ This phenomenon is thought to result from release of mycobacterial antigens after ATT, strengthening of host immune response, and decrease in immunosuppression mechanisms. ${ }^{10}$ Paradoxical worsening in ocular TB has been described both in immunocompromised and immunocompetent patients. ${ }^{5,11-13}$ We have earlier described four cases of paradoxical worsening in the eye, two of which occurred following ATT for extra-ocular TB. ${ }^{13}$ Gupta et al ${ }^{5}$ reported 12 patients with SLC ( $14 \%$ of 84 patients in the study), who developed progressive inflammation following initiation of ATT. This is less than half of the $29.2 \%$ (7 out of 24 patients) incidence seen in our patients with SLC. The median time of progression in SLC patients was 0.5 months in our study that is less than the 4 weeks noted in the earlier series. ${ }^{5}$ Anecdotal experience suggests that larger SLC lesions (representing a higher antigenic load) are more likely to develop such paradoxical worsening. Inadequate corticosteroid therapy $(<1 \mathrm{mg} / \mathrm{kg}$ body weight/day) during initiation of ATT may also lead to disease progression. All the SLC patients in our study, as in the earlier series, improved with increased immunosuppression and continued ATT. In addition, the role of rifampicin in reducing the bioavailability of corticosteroids also needs to be considered. Rifampicin has been shown to increase the plasma clearance of prednisolone by $45 \%$ and reduce drug bioavailability in tissues by $66 \% .{ }^{14}$ Because we did not consider this factor in deciding corticosteroid dosage, we may have undertreated the inflammatory component of the disease that led to progressive inflammation. Noncompliance to ATT can also lead to disease progression. In the present study, noncompliance, as well as drug toxicity, was monitored by the treating physician.

We also found paradoxical worsening in other forms of presumed ocular TB namely anterior uveitis, retinal vasculitis, multifocal choroiditis, and pan-uveitis. In addition, in four cases, we noted ocular inflammation at anatomical sites other than the presenting site. One patient with unilateral granulomatous anterior uveitis developed bilateral multifocal choroiditis, two developed episcleritis, and one patient developed optic neuritis. We speculate that this may be a result of multiple mycobacterial foci (and thereby antigenic stimulation) at various anatomical locations in the eye.

However, in case of intermediate uveitis, majority of patients with progressive inflammation (seven out of nine) did not respond to increase in corticosteroid therapy. In one patient, the diagnosis was revised to
Lyme's disease (and the patient responded to specific anti-microbial therapy), but in the remaining six, it remained undetermined. Five of these six patients resolved with therapeutic vitrectomy, whereas one patient had persistent inflammation till last follow-up. In an earlier series on intermediate uveitis from India, 5 out of 42 patients (11.9\%) who received ATT for presumed tubercular etiology had recurrent ocular inflammation, though its management was not described. ${ }^{15}$ We could not explain the high incidence of disease progression in our intermediate uveitis patients. Significantly, none of the intermediate uveitis patients with disease progression had associated focal chorio-retinal lesions. It is possible that the immunopathogenesis of intermediate uveitis is different from other forms of tubercular uveitis, and requires a different approach to management.

The role of 6-month ATT as against longer-duration therapy in causing progressive ocular inflammation is debatable. Although some studies have recommended a longer duration of 9-18 months of therapy, ${ }^{3,16}$ others have followed a 6-month ATT schedule. ${ }^{17}$ We followed the WHO recommendations for extra-pulmonary TB. ${ }^{8}$ Though a longer duration of 9-12 months is recommended for meningeal $\mathrm{TB}$, there is no evidence to suggest that the same should be followed for ocular TB as well. Recently, Ang et al ${ }^{16}$ reported that ATT of more than 9-month duration was superior to 6-9-month therapy in treatment of TB-associated uveitis. However, the sample size in this study was relatively small, and larger studies with longer follow-up are required to settle this debate.

Importantly, we could not detect drug resistance in our patients with disease progression. However, multi-drug resistance is less frequently seen in extra-pulmonary TB than in pulmonary TB. ${ }^{18}$ Also, in the absence of definitive ocular or systemic evidence (M. tuberculosis or its DNA) of $\mathrm{TB}$, it is not possible to diagnose drug resistance. Development of PCR-based diagnostic techniques may resolve this issue in future. Reinfection, though rare, can also cause disease progression, but, like drug resistance, remains difficult to investigate in ocular TB.

To summarize, our study provides a guideline to treating physicians to approach progressive ocular inflammation following ATT for presumed ocular TB. Most cases probably represent paradoxical worsening and resolve on escalation of corticosteroid therapy. In cases not responding to increased immunosuppression, targeted reinvestigations to rule out a nontubercular etiology and the possibility of drug interaction between rifampicin and corticosteroids need to be considered. However, the need for correct diagnosis at initial presentation itself, by detailed history and tailored laboratory investigations, remains vital. 


\section{Summary}

\section{What was known before}

- Beneficial effect of anti-tubercular therapy in TBassociated uveitis paradoxical worsening of ocular inflammation following anti-tubercular therapy.

What this study adds

- Incidence and clinical course of progressive ocular inflammation following anti-tubercular therapy in various clinical manifestations of presumed ocular TB Guidelines for identification and management of different forms of progressive ocular inflammation.

\section{Conflict of interest}

The authors declare no conflict of interest.

\section{References}

1 Bloom BR, Murray CJ. Tuberculosis: commentary on a reemergent killer. Science 1992; 257: 1055-1064.

2 Singh R, Gupta V, Gupta A. Pattern of uveitis in a referral eye clinic in north India. Indian J Ophthalmol 2004; 52: 121-125.

3 Bansal R, Gupta A, Gupta V, Dogra MR, Bambery P, Arora SK. Role of anti-tubercular therapy in uveitis with latent/manifest tuberculosis. Am J Ophthalmol 2008; 146: $772-779$.

4 Gupta A, Bansal R, Gupta V, Sharma A, Bambery P. Ocular signs predictive of tubercular uveitis. Am J Ophthalmol 2010; 149: 562-570.

5 Gupta V, Bansal R, Gupta A. Continued progression of tubercular serpiginous-like choroiditis after initiating antituberculosis treatment. Am J Ophthalmol 2011; 152: 857-863.

6 Jabs DA, Nussenblatt RB, Rosenbaum JT. Standardization of Uveitis Nomenclature (SUN) Working Group. Standardization of uveitis nomenclature for reporting clinical data. Results of the first international workshop. Am J Ophthalmol 2005; 140: 509-516.

7 Centers for Disease Control. Targeted tuberculin testing and treatment of latent tuberculosis infection. MMWR Morb Mortal Wkly Rep 2000; 49: 1-54.

8 World Health Organization. Treatment of Tuberculosis: Guidelines. 4th edn World Health Organization: Geneva, Switzerland, 2010, pp 95.

9 Ganesh SK, Roopleen, Biswas J, Veena N. Role of highresolution computerized tomography (HRCT) of the chest in granulomatous uveitis: a tertiary uveitis clinic experience from India. Ocul Immunol Inflamm 2011; 19: 51-57.

10 Afghani B, Lieberman JM. Paradoxical enlargement or development of intracranial tuberculomas during therapy: case report and review. Clin Infect Dis 1994; 19: 1092-1099.

11 Rathinam SR, Lalitha P. Paradoxical worsening of ocular tuberculosis in HIV patients after anti-retroviral therapy. Eye 2007; 21: 667-668.

12 Cheung CM, Chee SP. Jarisch-Herxheimer reaction: paradoxical worsening of tuberculosis chorioretinitis following initiation of antituberculous therapy. Eye 2009; 23: 1472-1473.

13 Basu S, Das T. Pitfalls in the management of TB-associated uveitis. Eye 2010; 24: 1681-1684.

14 McAllister WA, Thompson PJ, Al-Habet SM, Rogers HJ. Rifampicin reduces effectiveness and bioavailability of prednisolone. Br Med J (Clin Res Ed) 1983; 286: 923-925.

15 Parchand S, Tandan M, Gupta V, Gupta A. Intermediate uveitis in the Indian population. J Ophthalmic Inflamm Infect 2011; 1: 65-70.

16 Ang M, Hedayatfar A, Wong W, Chee SP. Duration of antitubercular therapy in uveitis associated with latent tuberculosis: a case-control study. Br J Ophthalmol 2012; 96: 332-336.

17 Gineys R, Bodaghi B, Carcelain G, Cassoux N, Boutin le TH, Amoura $\mathrm{Z}$ et al. QuantiFERON-TB gold cut-off value: implications for the management of tuberculosis related ocular inflammation. Am J Ophthalmol 2011; 152(3): 433-440.e1.

18 Peto HM, Pratt RH, Harrington TA, LoBue PA, Armstrong LR. Epidemiology of extrapulmonary tuberculosis in the United States, 1993-2006. Clin Infect Dis 2009; 49: 1350-1357. 